\title{
BMJ Open Costs associated with implementation of a strict policy for controlling spread of highly resistant microorganisms in France
}

Gabriel Birgand, ${ }^{1,2,3}$ Christophe Leroy, ${ }^{4}$ Simone Nerome, ${ }^{5}$ Liem Binh Luong Nguyen, ${ }^{6}$ Isabelle Lolom, ${ }^{3}$ Laurence Armand-Lefevre, ${ }^{7}$ Céline Ciotti, ${ }^{5}$ Bertrand Lecorre, ${ }^{6}$ Géraldine Marcade, ${ }^{8}$ Vincent Fihman, ${ }^{8}$ Marie-Hélène Nicolas-Chanoine, ${ }^{9}$ Camille Pelat, ${ }^{1,2}$ Anne Perozziello, ${ }^{10}$ Bruno Fantin, ${ }^{6}$ Yazdan Yazdanpanah, ${ }^{1,2,11}$ Jean-Damien Ricard, ${ }^{1,2,12}$ Jean-Christophe Lucet $^{1,2,3}$

To cite: Birgand G, Leroy C, Nerome S, et al. Costs associated with implementation of a strict policy for controlling spread of highly resistant microorganisms in France. BMJ Open 2016;6:e009029. doi:10.1136/bmjopen-2015009029

- Prepublication history for this paper is available online. To view these files please visit the journal online (http://dx.doi.org/10.1136/ bmjopen-2015-009029).

J-DR and J-CL contributed equally to the study.

Received 12 June 2015 Revised 23 October 2015 Accepted 27 October 2015

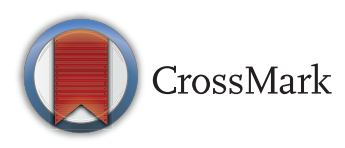

For numbered affiliations see end of article.

Correspondence to Dr Gabriel Birgand; gbirgand@gmail.com

\section{ABSTRACT}

Objective: To assess costs associated with implementation of a strict 'search and isolate' strategy for controlling highly drug-resistant organisms (HDRO).

Design: Review of data from 2-year prospective surveillance (01/2012 to 12/2013) of HDRO.

Setting: Three university hospitals located in northern Paris.

Methods: Episodes were defined as single cases or outbreaks of glycopeptide-resistant enterococci (GRE) or carbapenemase-producing Enterobacteriacae (CPE) colonisation. Costs were related to staff reinforcement, costs of screening cultures, contact precautions and interruption of new admissions. Univariate analysis, along with simple and multiple linear regression analyses, was conducted to determine variables associated with cost of HDRO management.

Results: Overall, 41 consecutive episodes were included, 28 single cases and 13 outbreaks. The cost (mean \pm SD) associated with management of a single case identified within and/or $48 \mathrm{~h}$ after admission was $€ 4443 \pm 11552$ and $€ 11445 \pm 15743$, respectively $(p<0.01)$. In an outbreak, the total cost varied from $€ 14864 \pm 17734$ for an episode with one secondary case $(€ 7432 \pm 8867$ per case) to $€ 136525 \pm 151231$ (€12 845 \pm 5129 per case) when more than one secondary case occurred. In episodes of single cases, contact precautions and microbiological analyses represented $51 \%$ and $30 \%$ of overall cost, respectively. In outbreaks, cost related to interruption of new admissions represented $77-94 \%$ of total costs, and had the greatest financial impact $\left(R^{2}=0.98\right.$, $p<0.01$ ).

Conclusions: In HDRO episodes occurring at three university hospitals, interruption of new admissions constituted the most costly measure in an outbreak situation.

\section{Strengths and limitations of this study}

Multicentre study to estimate costs of a strict policy for controlling highly drug-resistant organisms (HDRO) spread with data collected prospectively, enabling detailed cost analysis in a large panel of situations.

- Provides a basis for minimising the financial burden of a 'search and isolate' strategy. Early identification of patients suspected of being colonised and rapid implementation of contact precautions represented the least expensive scenario.

- The study raises the question of having, or creating, dedicated areas, thus enabling continuity of care together with cost savings.

- The study did not include: loss of revenue due to systematic placement of colonised patients in a single room, costs linked to prolongation of the hospital stay of case patients and time spent by the infection control team in managing episodes.

- Cost estimations were based on local levels of hospital reimbursement.

\section{INTRODUCTION}

Hospitals are increasingly plagued by highly drug-resistant organisms (HDRO) to antimicrobials. ${ }^{1}$ These HDRO include carbapenemase-producing Enterobacteriaceae (CPE) and glycopeptide-resistant enterococci (GRE). In France, the prevalence of GRE and carbapenemase-producing Klebsiella pneumoniae isolated from blood cultures was $0.8 \%$ and $0.5 \%$, respectively, ${ }^{2}$ in 2012 .

In France, guidelines based on a 'search and isolate' strategy have been issued for the control of emerging HDRO. ${ }^{3}$ They are based 
on two assumptions: (1) most HDRO-positive patients are asymptomatic carriers with high risk of spreading before outbreak identification and (2) standard or contact precautions do not reliably halt HDRO transmission in all circumstances.

Infection control measures are gradually implemented according to risk analysis. In case of immediate enforcement of strict contact precautions (identification of a colonised patient on hospital admission, notably if repatriated or recently hospitalised abroad in the past 12 months), weekly cross-sectional screening of ward patients is recommended, with no additional control measures. ${ }^{34}$

In an outbreak situation, that is, with at least one secondary case, measures are upgraded and consist of a strict 'search and isolate' strategy as follows: (1) HDRO-positive patients are cohorted and cared for by dedicated staff; (2) secondary cases are detected via repeated rectal sampling of contact patients, that is, patients cared for by the same nursing staff as the HDRO-positive patient; (3) contact patients are cohorted and cared for by dedicated staff until three weekly screening tests are negative; (4) HDRO-positive and contact patients are discharged home whenever possible; and (5) the ward with the HDRO-positive patients neither transfers patients to other wards or healthcare facilities (HCFs) nor admits new patients until after three negative weekly screening tests of contact patients.

These strict recommendations are difficult to implement and require additional human and material resources. Moreover, interruption of admission to and transfer from the involved ward leads to a decrease in hospital medical service utilisation and therefore a loss of hospital income. ${ }^{5}$ Costs associated with each different epidemiological situation and the determinants of these costs are not known. The purpose of this study was to assess costs associated with implementation of national recommendations for controlling HDRO spread in three university hospitals and to identify determinants of these costs.

\section{PATIENTS AND METHODS}

\section{Setting}

This study was performed in a French university healthcare group located in northern Paris, the 950-bed Bichat-Claude Bernard Hospital, the 470-bed Beaujon Hospital and the 490-bed Louis-Mourier Hospital, providing primary, tertiary and long-term care with a large panel of surgical and medical specialties. This trust takes part of a public health institution (AP-HP) representing $10 \%$ of all public hospital beds in France. These three hospitals are situated in the highly exposed area with a high proportion of patients originating from a foreign country. None of these hospitals has a dedicated ward for housing/regrouping case patients. Case patients were therefore admitted to the ward matching their pathology. In outbreak situations, however, case patients from different wards could be housed in the ward with the highest case number.

\section{Design and data collection on resources used}

We reviewed data from a 2-year prospective surveillance of HDRO occurrence (01/2012-12/2013). We defined an episode as consisting of new identification of HDRO in a clinical or screening sample, unrelated to previous situations. An outbreak was defined as at least two CPE cases (ie, one index case and at least one secondary case among the contact patients) occurring in a given hospital, with a clear epidemiological link (stay during the same period of time in the same unit) and involving an indistinguishable CPE strain based on the species, antibiotic susceptibility and resistance gene. We distinguished four types of episodes involving CPE or GRE strains, from simple situations with low epidemic risk to complex situations with confirmed outbreaks: (1) a single case suspected within $48 \mathrm{~h}$ after hospital admission; (2) a single case suspected more than $48 \mathrm{~h}$ after hospital admission; (3) an outbreak with only one secondary case; and (4) an outbreak with more than one secondary case.

For each episode, data were prospectively collected, including characteristics of the epidemic (type of HDRO and resistance mechanism, type of ward, dates of admission and discharge of case patients, date of positive results and implementation of contact precautions, number of contact patients); human resources (nursing staff reinforcement allocated to a ward during an episode, either for cohorting colonised patients, ie, placing the patient in a dedicated location on the ward with dedicated healthcare workers (HCWs), or for decreasing the workload of the unit by globally increasing the nurse-to-patient ratio); material for the three weekly screening protocol and patient care; and duration of interruption of new admissions.

\section{Cost analysis}

Costs were considered from a hospital perspective. For human resources, staff reinforcement was calculated on the basis of the number of supplementary hours put in by nurses and nursing assistants on the basis of their hourly salary (table 1 ). The cost calculation was performed until the date of analysis, 9 months after the end of the last episode. Costs associated with readmission of contact patients after this date were not considered.

For the laboratory sector, methods for detecting CPE and GRE in screening samples have been described elsewhere. ${ }^{8}{ }^{9}$ Unit costs for resources used to screen were computed on the basis of use of the following resources: selective chromogenic plates or PCR-based method, identification tests and susceptibility tests, depending on the above-described situations. Personnel costs for laboratory tests were calculated on the basis of the hourly salary of a senior staff member and the estimated time required for each step. Unit cost of PCR screening included acquisition of the GeneXpert machine 
Table 1 Methods of cost analyses

\begin{tabular}{|c|c|c|}
\hline Type & Variables collected & Value \\
\hline \multirow[t]{5}{*}{ Loss of income } & Number of hospital bed-days lost & \\
\hline & Mean cost billed per hospital-day per specialty & \\
\hline & Medical units & $€ 335$ to $€ 601$ \\
\hline & Surgical units & $€ 306$ to $€ 940$ \\
\hline & $\mathrm{ICU}$ & $€ 609$ to $€ 2078$ \\
\hline \multirow[t]{2}{*}{ Staff reinforcement } & Cost of $1 \mathrm{~h}$ of a nursing assistant & $€ 24.6$ \\
\hline & Cost of $1 \mathrm{~h}$ of a nurse & $€ 30.5$ \\
\hline \multirow[t]{6}{*}{ Cost of micro-analysis ${ }^{5}$} & Negative culture for GRE & $€ 13.9$ \\
\hline & Cepheid Xpert vanA/vanB & $€ 37.3$ \\
\hline & Negative culture for CPE & $€ .7$ \\
\hline & Negative culture for CPE, + for ESBLPE & $€ 21$ \\
\hline & Positive culture for CPE & $€ 115$ \\
\hline & Positive culture for GRE strain & $€ 117.8$ \\
\hline \multirow[t]{6}{*}{ Cost of contact precautions ${ }^{6}$} & Cumulative number of hospital-days, HDRO patients & $€ 0.05 /$ pair \\
\hline & Cost of gloves & $€ 0.3$ each \\
\hline & Cost of gowns & $€ 0.26$ \\
\hline & Cost of nursing contact (1 min) Papia et al ${ }^{7}$ & $€ 30$ \\
\hline & Number of patient contacts per day & $€ 18.5$ \\
\hline & Cost per HDRO patient per day & \\
\hline
\end{tabular}

(Cepheid, Sunnyvale, California, USA) and Xpert vanA/ van $\mathrm{B}$ test cartridges and performing cultures for GRE on a vanA/vanB-positive sample or samples without PCR results (invalid tests). ${ }^{5}$ Cost of contact precautions included that of gloves and gowns used for case patients, assuming an average of 30 patient contacts per day of isolation and nursing costs for additional time to donning and discarding gloves and gown $(1 \mathrm{~min}){ }^{6}$

Finally, to estimate the decrease in hospital service use, we first computed the difference between admission capacity, assuming $100 \%$ bed occupancy, and the number of patients admitted when an HDRO-positive patient was identified in the ward. Next, we estimated costs attributable to decreased occupancy, by multiplying the number of missed patient-days by the mean cost of a hospital-day, depending on the type of pathology and the ward. According to the French reimbursement system, the mean cost per hospital-day was the total cost related to hospital stay in the previous year in the affected ward divided by the number of patient-days. ${ }^{10}$ Total cost related to missed hospital-days in a ward was estimated using the French diagnosis-related group system according to which patients are classified into statistically and clinically homogeneous groups on the basis of their clinical and demographic data.

\section{Statistical analysis}

Categorical independent variables were described using proportions and continuous variables via medians and 25th-75th centiles. For costs, means with SD were used to take into account outliers and data dispersion. Univariate comparisons used a Wilcoxon rank or $\chi^{2}$ test as required. Statistics on categorical variables were based on two-way analysis of variance (ANOVA). After univariate analysis, simple and multiple linear regression analyses were carried out, with overall cost as the dependent variable, to determine those costs most strongly associated with the overall financial burden. The overall percentage of explained variance of the model was described by the adjusted $\mathrm{R}^{2}$ of observed costs. Predictive values of models built were tested using the method of 'Leave One Out Cross-Validation' (jackknife). ${ }^{11}$ This method assesses the predicted costs in one episode based on the model built with all other episodes. We analysed observed versus predicted costs for all episodes, and specifically for outbreaks, by giving the mean and median predictive error per episode and the mean relative predictive error. Statistical analyses were done with R software, V.2.15.2.

\section{Ethics committee approval}

Owing to the observational and blinded nature of the study, the institutional review board of the Bichat-Claude Bernard Hospital waived the requirement for informed consent. According to this statement, written consents of patients were not collected. Patient information was de-identified by attributing a number.

\section{RESULTS}

\section{Characteristics of HDRO episodes}

Overall, we observed 41 HDRO episodes (34 at Bichat-Claude Bernard, 6 at Beaujon and 1 at Louis-Mourier Hospital), with a total of 113 colonised or infected patients (table 2).

We observed 24 episodes in 2012 and 17 in 2013. Index cases were colonised with GRE in 20 (49\%) 
Table 2 Characteristics of episodes with highly resistant organisms according to type of episode

\begin{tabular}{|c|c|c|c|c|c|}
\hline $\begin{array}{l}\text { Description of episode } \\
\text { characteristics }\end{array}$ & $\begin{array}{l}\text { Total } \\
\mathrm{N}=41\end{array}$ & $\begin{array}{l}\text { A single case } \\
\text { (suspicion } \leq 48 \mathrm{~h} \\
\text { after admission) } \\
\mathrm{N}=14\end{array}$ & $\begin{array}{l}\text { A single case } \\
\text { (suspicion }>48 \mathrm{~h} \\
\text { after admission) } \\
\mathrm{N}=14\end{array}$ & $\begin{array}{l}\text { Episode with } \\
1 \text { secondary } \\
\text { case } \\
\mathrm{N}=6\end{array}$ & $\begin{array}{l}\text { Episode with } \\
>1 \text { secondary } \\
\text { case } \\
\mathrm{N}=7\end{array}$ \\
\hline \multicolumn{6}{|l|}{ Number of episodes per hospital, n (\%) } \\
\hline Bichat-Claude Bernard & $34(83)$ & $10(71)$ & $13(93)$ & $6(100)$ & $5(70)$ \\
\hline Beaujon & $6(15)$ & $4(29)$ & $1(7)$ & 0 & $1(15)$ \\
\hline Louis-Mourier & $1(3)$ & 0 & 0 & 0 & $1(15)$ \\
\hline \multicolumn{6}{|l|}{ Year, n (\%) } \\
\hline 2012 & $24(58)$ & $6(43)$ & $8(57)$ & $6(100)$ & $4(57)$ \\
\hline \multirow{2}{*}{\multicolumn{6}{|c|}{ Type of HDRO, n (\%) }} \\
\hline & & & & & \\
\hline GRE & $20(49)$ & $3(21)$ & $6(43)$ & $5(83)$ & $6(86)$ \\
\hline CPE & $19(46)$ & $10(71)$ & $8(57)$ & $1(17)$ & 0 \\
\hline GRE + CPE & $2(5)$ & $1(8)$ & 0 & 0 & $1(14)$ \\
\hline \multicolumn{6}{|l|}{ Type of ward at identification, $\mathrm{n}(\%)$} \\
\hline ICU & $7(17)$ & $4(29)$ & $2(14)$ & $1(17)$ & 0 \\
\hline Medical & $23(56)$ & $8(57)$ & $6(43)$ & $3(50)$ & $6(86)$ \\
\hline Surgical & $10(24)$ & $2(14)$ & $6(43)$ & $2(33)$ & 0 \\
\hline Rehabilitation & $1(3)$ & 0 & 0 & 0 & $1(14)$ \\
\hline $\begin{array}{l}\text { Time from admission to suspicion } \\
\text { (screening), days, median (IQR) }\end{array}$ & $4(1-26)$ & $0(0-1)$ & $12.5(5-33)$ & $14(4-26)$ & $42(3-75)$ \\
\hline $\begin{array}{l}\text { Time from admission to HDRO+ } \\
\text { result, days, median (IQR) }\end{array}$ & $6(3-26)$ & $0.5(0-3)$ & $12.5(5-33)$ & $14(7-26)$ & $42(6-75)$ \\
\hline $\begin{array}{l}\text { Number of contact patients, median } \\
\text { (IQR); minimum-maximum }\end{array}$ & $32(13-65)$ & $\begin{array}{l}5(0-21) \\
0-65\end{array}$ & $\begin{array}{l}34(19-76) \\
0-260\end{array}$ & $\begin{array}{l}48.5(32-53) \\
19-262\end{array}$ & $\begin{array}{l}66(53-152) \\
48-237\end{array}$ \\
\hline $\begin{array}{l}\text { Number of secondary cases, } \\
\text { median (IQR); minimum-maximum }\end{array}$ & - & 0 & 0 & 1 & 3 (2-22); 2-29 \\
\hline $\begin{array}{l}\text { Suspension of admissions, days } \\
\text { median (IQR); minimum-maximum }\end{array}$ & $0(0-3)$ & $0(0-0) ; 0-10$ & $1(0-3) ; 0-7$ & $3(0-3) ; 0-7$ & $8(6-12) ; 0-62$ \\
\hline
\end{tabular}

episodes, CPE in $19(46 \%)$ episodes and with both HDRO in $2(5 \%)$ episodes. HDRO were cultured from clinical samples in $13(12 \%)$ patients, 9 with GRE and 4 with CPE $(\mathrm{p}=0.85)$. Among the 41 episodes, $14(34 \%)$ were single cases suspected within $48 \mathrm{~h}$ after admission; $14(34 \%)$ were single cases suspected more than $48 \mathrm{~h}$ after admission; $6(15 \%)$ were with one secondary case; and $7(17 \%)$ were outbreaks with more than one secondary case. Patients colonised or infected with GRE were single cases in 10/22 cases and generated 12 outbreaks (among which one carried both GRE and CPE). These outbreaks resulted in a median of one secondary case (IQR 0-2; range 0-29). Episodes with CPE were single cases in 18/19 situations and with one secondary case in the remaining episode. The difference in the number of secondary cases was significant between GRE and CPE $(p<0.01)$. The affected wards included medical (23 episodes), surgical (10 episodes), intensive care (7 episodes) and rehabilitation (one episode) wards.

The median time from hospital admission to suspicion of a first case was 4 days (IQR 1-26). It was $1(0-8)$ and 14 days (3-42) for CPE and GRE, respectively ( $p=0.01)$. This duration was significantly longer in outbreak situations, 21 (4-62) vs 2 (0-12.5) days in single cases
( $p<0.01)$. In eight episodes, the suspected patient was placed in a contact precaution state on hospital admission, the risk of cross transmission was considered low and contact patients were not followed. For the 33 other episodes, the median number of contact patients was 32 (IQR 13-65). The number of contact patients was higher for GRE than for CPE episodes, 52 (15-76) and 24 (0-37), respectively, $(\mathrm{p}=0.06)$.

The median duration of episodes was 3 days (IQR 0 10). Interruption of new admissions was decided for 20 episodes. The median duration of interruption in outbreak situations ( $\geq 1$ secondary case) was 4.5 days (IQR $1.5-8$, range $0-62$ ). This median duration of interruption was significantly higher if case patients were suspected and isolated more than $48 \mathrm{~h}$ after admission (2 days) than for suspected patients identified at hospital admission ( 1 day) $(p=0.02)$ and for outbreaks with only one secondary case ( 3 days) as compared to outbreaks with more than one secondary case ( 16 days) $(\mathrm{p}=0.02)$.

\section{Costs associated with HDRO episodes}

Concerning human resources, the nursing staff was reinforced in 16 episodes, among which $10(62 \%)$ were outbreak episodes. Nursing assistants represented the main 
reinforced staff category, with a mean of 61 supplementary hours per episode (range 0-1603). Nurses were requested in 15 episodes, with a mean number of 38 supplementary hours. The mean cost associated with staff reinforcement was €2686 (SD €8861), varying from $€ 0$ to $€ 55081$ (table 3).

For laboratory resources, the median number of screening samples performed per episode was 69 (IQR 27-119), with 110 (IQR 66-152) in GRE episodes and 46 (IQR 13-74) in CPE episodes. Mean costs of microbiological analysis were €2050 (SD €3428) and €3423 (SD €4479) for GRE episodes and €742 (SD €872) for CPE episodes $(\mathrm{p}<0.01)$.

The median duration of contact precautions for HDRO-colonised patients was 33 (IQR 17-65) days. The mean cost of protective equipment used for contact isolation was $€ 931$ (SD €1022).

In wards affected by an episode of HDRO, the duration of interruption of admissions ranged from 0 to 694 patient bed-days according to the episode, with a mean varying from 7 patient bed-days for episodes with a single case identified at admission to 241 patient bed-days in case of outbreak with more than one secondary case. The mean cost associated with interruption of admissions was estimated at €25 242 (SD €67 297), varying from $€ 0$ to $€ 348468$ for the largest outbreak. In single HDRO cases, the mean cost associated with interruption of admissions for the episode was significantly higher when the case patient was suspected $>48 \mathrm{~h}$ after admission (€9666) than when it was suspected $<48 \mathrm{~h}$ $(€ 2493, \mathrm{p}<0.01)$. In the 13 outbreaks, the mean cost associated with interruption of admissions for the episode was $€ 66516$ (SD $€ 109557$ ), varying from $€ 0$ in three situations with one secondary case to $€ 348468$ with 29 secondary cases. The mean cost associated with interruption of admissions was $€ 44020$ for GRE episodes and $€ 6834$ for CPE episodes ( $\mathrm{p}=0.18$ ).

The overall mean cost of infection control measures was $€ 4443$ in a single case identified within $48 \mathrm{~h}$ after admission. Mean costs were higher if a single case was identified more than $48 \mathrm{~h}$ after the admission, at $€ 11445(\mathrm{p}<0.01)$. In an outbreak situation, the mean cost varied from $€ 14864$ (SD $€ 17734$ ) for an episode with one secondary case to $€ 136525$ (SD $€ 151231$ ) for outbreaks with at least two secondary cases. The mean cost per case was $€ 7432$ (SD $€ 8867$ ) in episodes with one secondary case and $€ 12845$ (SD €5129) in other outbreak episodes $(\mathrm{p}<0.01)$.

\section{Analysis by category of cost}

Overall, the cost associated with interruption of admissions represented the most expensive category, with an average of $38 \%$ (range $0-97 \%$ ) of total cost per episode, followed by microbiology testing 29\% (0-100\%), contact precautions $27 \%$ and staff reinforcement $6 \%$ per episode (figure 1). When outbreaks had one secondary case, cost associated with interruption of admissions represented $53 \%$ of total cost per episode; this proportion increased to $74 \%$ when outbreaks had more than one secondary case. In episodes with a single case suspected within the first $48 \mathrm{~h}$ of admission, contact precautions and microbiological analyses represented 53\% and $34 \%$ of average overall cost per episode, respectively. When we aggregated costs for all episodes, the interruption of activity represented $81.7 \%$ of the overall cost, followed by human resources $8.7 \%$, microbiology $6.6 \%$ and contact precautions $3 \%$.

Linear regression analysis was performed to assess cost determinants using data from the 41 episodes. Individually, cost associated with interruption of admissions was the highest item affecting the cost of infection control strategies $\left(\mathrm{R}^{2}=0.98, \mathrm{p}<0.01\right)$, followed by microbiological analyses $\left(R^{2}=0.76, p<0.01\right)$, staff reinforcement $\left(\mathrm{R}^{2}=0.59, \mathrm{p}<0.01\right)$ and contact precautions $\left(\mathrm{R}^{2}=0.25\right.$, $\mathrm{p}<0.01)$. The linear model, including the duration of interruption of new admissions as an independent variable, predicted the overall cost of episodes, with a median error of $€ 3394$ (IQR $€ 704-€ 15942$ ), or $62 \%$ of median overall cost. When restricting analysis to the seven outbreak episodes with at least two secondary cases, the same model more accurately predicted overall cost, with a median error of $€ 19038$ (IQR $€ 16056$ $€ 69486$ ), or $27 \%$ of the median overall cost per episode. We used single and multiple linear regression to predict overall cost, using all potential explicative variables, individually or combined. None of the models built accurately fit the data.

\section{DISCUSSION}

This study was performed to assess the financial burden of implementation of a strict national policy to control the spread of HDRO. The mean cost per episode was measured at $€ 4443$ per episode for single cases isolated at admission to $€ 136525$ for outbreaks with at least two secondary cases. The mean cost per case varied from $€ 4443$ for a single case to $€ 12845$ in outbreak situations. Interruption of admissions was the most costly measure in an outbreak situation.

To the best of our knowledge, this is the first multicentre study to estimate costs of a strict policy for controlling HDRO spread. Data were collected prospectively, enabling detailed cost analysis in a large panel of situations. Previous studies mainly focused on GRE, and primarily assessed costs related to an outbreak situation, ${ }^{12-14}$ infection, prolonged length of stay ${ }^{15-17}$ or implementation of surveillance. ${ }^{18-21}$ Studies focusing on cost associated with outbreak situations measured the overall financial burden, varying from $€ 4161$ to $€ 40131$ per case. ${ }^{12-1421}$ Methods used were variable, with approximate measures and occasional missed critical cost categories.

Antibiotic resistance has become a worldwide concern, and a recent WHO report warned of a 'post-antibiotic era'. Strict French national strategy appeared to be effective in controlling the spread of HDRO, as illustrated by European Antimicrobial Resistance 
Table 3 Resources used in, and costs associated with, episodes of highly resistant organisms per type of episodes

\begin{tabular}{|c|c|c|c|c|c|c|c|c|c|c|}
\hline & \multicolumn{2}{|l|}{$\begin{array}{l}\text { Total } \\
\mathrm{N}=41\end{array}$} & \multicolumn{2}{|c|}{$\begin{array}{l}\text { One single case } \\
\text { (suspicion } \leq 48 \mathrm{~h} \text { after } \\
\text { admission) } \\
\mathrm{N}=14\end{array}$} & \multicolumn{2}{|c|}{$\begin{array}{l}\text { One single case } \\
\text { (suspicion }>48 \mathrm{~h} \text { after } \\
\text { admission) } \\
\mathrm{N}=14\end{array}$} & \multicolumn{2}{|c|}{$\begin{array}{l}\text { Episode with } 1 \\
\text { secondary case } \\
\mathrm{N}=6\end{array}$} & \multicolumn{2}{|c|}{$\begin{array}{l}\text { Episode with }>1 \\
\text { secondary case } \\
\mathrm{N}=7\end{array}$} \\
\hline & $\begin{array}{l}\text { Median } \\
\text { (IQR) }\end{array}$ & $\begin{array}{l}\text { Minimum- } \\
\text { maximum }\end{array}$ & $\begin{array}{l}\text { Average } \\
\text { (SD) }\end{array}$ & $\begin{array}{l}\text { Minimum- } \\
\text { maximum }\end{array}$ & $\begin{array}{l}\text { Average } \\
\text { (SD) }\end{array}$ & $\begin{array}{l}\text { Minimum- } \\
\text { maximum }\end{array}$ & $\begin{array}{l}\text { Average } \\
\text { (SD) }\end{array}$ & $\begin{array}{l}\text { Minimum- } \\
\text { maximum }\end{array}$ & $\begin{array}{l}\text { Average } \\
\text { (SD) }\end{array}$ & $\begin{array}{l}\text { Minimum- } \\
\text { maximum }\end{array}$ \\
\hline Loss of income, $€ 1000$ s & $25.2(67.3)$ & $0-348.5$ & $2.5(9.3)$ & $0-34.9$ & $9.6(15.7)$ & $0-54.9$ & $10.2(16.2)$ & $0-40.6$ & $115(134)$ & $1.5-348$ \\
\hline Loss hospital bed-days & 35 (88.7) & $0-520$ & $7.4(27.8)$ & $0-104$ & $13.6(23.2)$ & $0-90$ & $19.2(28.3)$ & $0-67$ & $165(182)$ & $5-520$ \\
\hline Staff reinforcement, $€ 1000$ s & $2.7(8.8)$ & $0-55.1$ & $0.77(2.3)$ & $0-8.4$ & $0.3(0.6)$ & $0-1.9$ & $0.7(1.1)$ & $0-2.9$ & $12.9(18.9)$ & $0.45-55.1$ \\
\hline Hours of assistant nurses & $61.4(252.7)$ & $0-1603$ & $15.7(58.7)$ & $0-219.5$ & $4.4(11.4)$ & $0-42$ & $9.3(11.9)$ & $0-30$ & $311(574)$ & $0-1603$ \\
\hline Hours of nurses & $38.5(93.5)$ & $0-512$ & $12.8(32.7)$ & $0-98$ & $5.9(14.2)$ & $0-48$ & $16,1(27.6)$ & $0-71$ & $174(169)$ & $7.5-512$ \\
\hline $\begin{array}{l}\text { Cost of microbiological analysis, } \\
€ 1000 \text { s }\end{array}$ & $2.0(3.4)$ & $0-19.6$ & $0.53(0.49)$ & $0-1.5$ & $0.9(0.64)$ & $0.13-2.2$ & $2.7(1.7)$ & $0.87-5.5$ & $6.7(6.3)$ & $2.3-19.6$ \\
\hline \multicolumn{11}{|l|}{ For GRE strains, number of } \\
\hline Negative culture & $59.9(101.6)$ & $0-426$ & $39.7(33.7)$ & $0-75$ & $65(52)$ & $10-150$ & $104(95.7)$ & $17-263$ & $198(156)$ & $76-426$ \\
\hline Cepheid Xpert vanA/vanB & $18.4(45.4)$ & $0-279$ & $0.8(2.9)$ & $0-11$ & $4.1(8.8)$ & $0-29$ & 32.7 (21.9) & $0-62$ & $70.4(92.8)$ & $20-279$ \\
\hline Positive culture & $2.2(6.2)$ & 0-29 & $0.2(0.5)$ & $0-1$ & $0.6(0.5)$ & $0-1$ & $2(0)$ & $2-2$ & $10.7(12.2)$ & $2-29$ \\
\hline \multicolumn{11}{|l|}{ For CPE strains, number of: } \\
\hline Negative culture & $20.5(35.5)$ & $0-137$ & $27(44)$ & $0-137$ & $49(35)$ & $0-112$ & 70 & $70-70$ & 102 & $102-102$ \\
\hline $\begin{array}{l}\text { Negative culture, + for } \\
\text { ESBLPE }\end{array}$ & $4.6(10.3)$ & $0-61$ & $15.5(19.1)$ & $1-61$ & $7.7(4.2)$ & $4-17$ & 5 & $5-5$ & - & - \\
\hline Positive culture & $0.12(0.39)$ & $0-2$ & $0.1(0.3)$ & $0-1$ & $0.2(0.4)$ & $0-1$ & 2 & $2-2$ & 0 & $0-0$ \\
\hline Cost of contact isolation, $€ 1000$ s & $0.93(1.0)$ & $0-4.7$ & $0.63(0.74)$ & $0.1-3.1$ & $0.58(0.57)$ & $0-1.8$ & $1.18(0.44)$ & $0.61-1.79$ & $1.99(1.74)$ & $0.49-4.69$ \\
\hline $\begin{array}{l}\text { Cumulative LOS of HDRO } \\
\text { patients }\end{array}$ & $49.4(55.7)$ & $0-254$ & $34.3(40.3)$ & $7-166$ & $31.8(30.7)$ & $0-98$ & 64 (23.9) & $33-97$ & $111.5(102.7)$ & $27-254$ \\
\hline Overall cost, $€ 1000$ s & $30.9(77.2)$ & $0.3-370.7$ & $4.44(11.5)$ & $0.3-44.3$ & $11.4(15.7)$ & $0.6-57.2$ & $14.8(17.7)$ & $1.4-45.9$ & $136.5(151.2)$ & $16.7-370.7$ \\
\hline Cost per case, $€ 1000$ s & $8.7(12.2)$ & $0.3-57.2$ & $4.44(11.5)$ & $0.3-44.3$ & $11.4(15.7)$ & $0.6-57.2$ & $7.4(8.8)$ & $0.7-22.9$ & $12.8(5.1)$ & $4.1-12.3$ \\
\hline
\end{tabular}



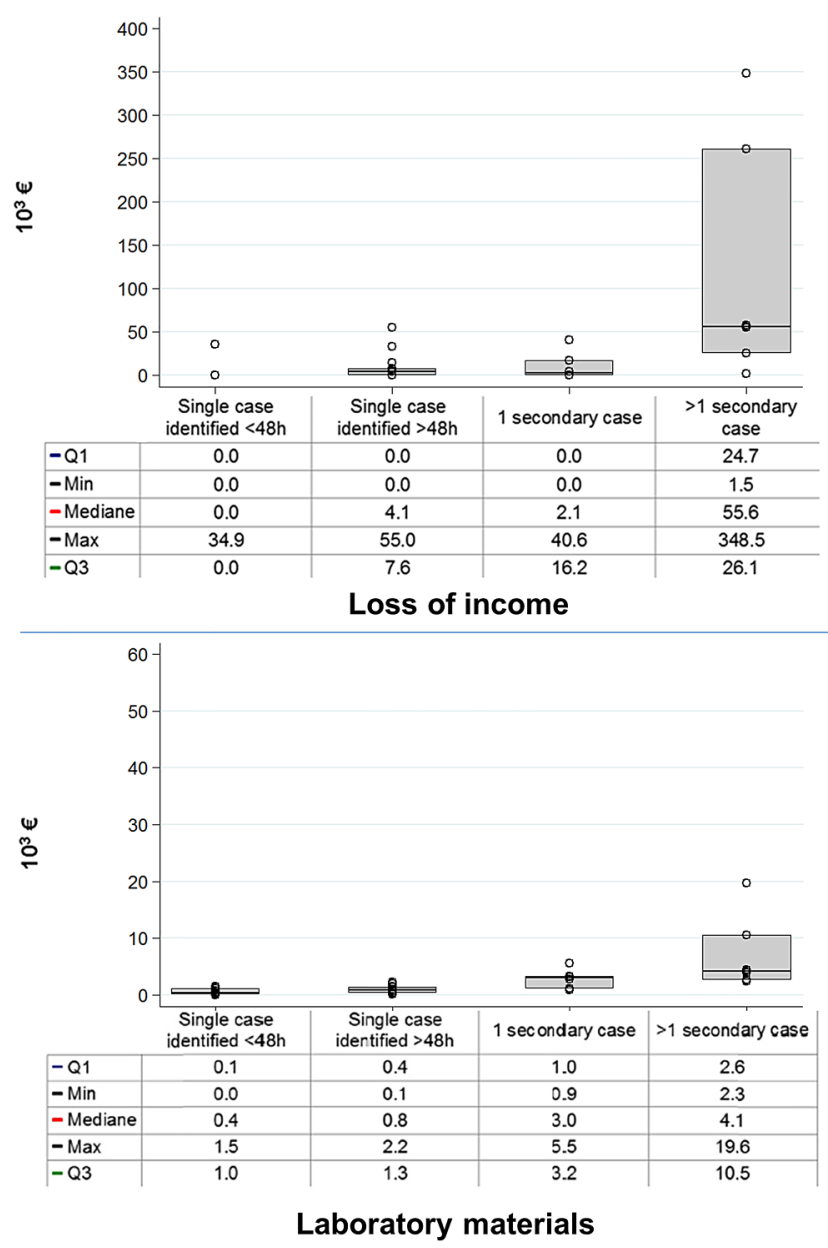

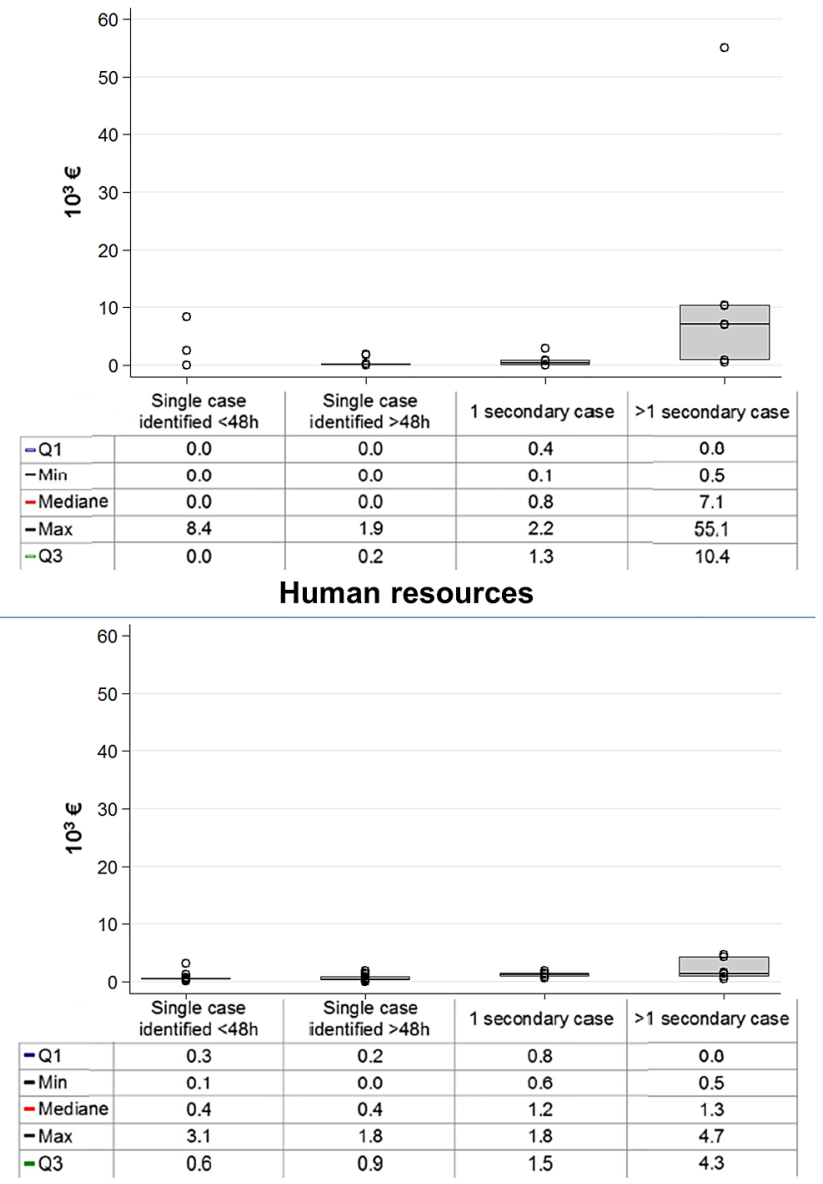

Equipment for contact precautions

Figure 1 Cost distribution per category of resource and type of episode. Min, minimum; max, maximum.

Surveillance System data and results from large hospital networks. ${ }^{2}$ 22-24 These guidelines exclude multidrug resistance organisms requiring basic contact precautions by the fact that they have become endemic (methicillinresistant Staphylococcus aureus) or pandemic (extended spectrum $\beta$-lactamase-producing Enterobacteriacae), making the search and destroy strategy useless. In emerging situations, we can assume that applying a search and isolated strategy for the control of these organisms would lead to comparable costs. Despite a small proportion of HDRO-positive clinical samples and the fact that very few were infected, this control strategy may be justified by the high colonised-to-infected ratio, with possible spread from unidentified colonised patients. However, these recommendations are costly, difficult to implement on a practical basis and require human/laboratory resources and occasional need for interruption of admissions. This study provides a basis for minimising the financial burden of a 'search and isolate' strategy.

In our study, early identification of patients suspected of being colonised and rapid implementation of contact precautions represented the least expensive scenario. In this context, ward activity was usually maintained, with costs mainly due to staff reinforcement and laboratory techniques. In situations with delayed identification, suspension of admissions was often decided pending results of screening of contact patients. Along this line, guidelines were issued in order to promptly identify, screen and implement strict contact precautions for patients recently hospitalised in a foreign country. ${ }^{325}$

In outbreak situations, suspension of admissions was the most expensive measure, with mean costs of every secondary case estimated at $€ 12845$, whereas costs due to human resources were lower. This study raises the question of having, or creating, dedicated areas, thus enabling continuity of care together with cost savings. A literature review did not find any study that performed cost-effectiveness/benefit or savings analysis of strict measures for controlling HDRO spread in outbreak situations. This underlines the need for further studies on cost-effectiveness of different strategies to control HDRO dissemination and optimise both the financial and medical burden of recommendations.

Our study had limitations. First, we did not include potential loss of revenue due to systematic placement of colonised patients in a single room. Like other French HCF, the three hospitals possess a high rate of single rooms for patient isolation or privacy. ${ }^{26}$ Hence, we assumed that a single room was standard in the affected unit. Second, costs were estimated on the basis of local 
levels of hospital reimbursement. Costs of suspension of admissions would be much higher in hospitals with higher daily costs. However, a quick review shows that costs per bed-days are very similar to those found in the literature. ${ }^{27-30}$ Moreover, the presentation with a proportion of the overall cost by category allows a clear interpretation. Third, we did not consider costs linked to prolongation of the hospital stay of case patients. We had previously estimated the average prolongation at 23 days, representing $€ 6981$ to $€ 47800$ per case. ${ }^{31}$ In this study, it was not possible to precisely determine the prolongation of hospital stay, which would have been based on a subjective ward physician estimate. Fourth, we did not measure time spent by the infection control team in managing episodes. Fifth, the setting of this study (three hospitals in one country) imposes the caution regarding the generalisability of crude costs. However, distributions of expenses should remain approximately the same whatever the hospital and the country. Sixth, the loss of activity was estimated on the basis of a $100 \%$ bed occupancy. This assumption appears to be contestable, but the proportion of free bed-days was so small that we considered them as negligible. Finally, no statistical model built fit the data, mainly because of the heterogeneity of situations and control measures. Specifically, loss of income varied from $€ 0$ to $€ 54976$ for episodes with single cases and represented the most heterogeneous variable, directly linked to the context and risk assessment and control measures decided/set by the infection control team.

In conclusion, cost analysis of a large number of episodes showed that suspension of admission was the most costly measure in an outbreak situation. Further studies are needed to assess the cost-effectiveness of cohorting to control HDRO spread. Early identification and implementation of contact precautions may lead to major cost savings in the context of a strict HDRO policy.

\footnotetext{
Author affiliations

${ }^{1}$ INSERM, IAME, UMR 1137, Paris, France

${ }^{2}$ Univ Paris Diderot, IAME, UMR 1137, Sorbonne Paris Cité, Paris, France

${ }^{3}$ Infection Control Unit, AP-HP, Hôpital Bichat-Claude Bernard, Paris, France

${ }^{4}$ Emergency Department, AP-HP, Hôpital Louis Mourier, Colombes, France

${ }^{5}$ Infection Control Unit, AP-HP, Hôpital Beaujon, Clichy, France

${ }^{6}$ Internal Medicine Department, AP-HP, Hôpital Beaujon, Clichy, France

${ }^{7}$ Bacteriology Laboratory, AP-HP, Hôpital Bichat-Claude Bernard, Paris, France

${ }^{8}$ Infection Control Unit, AP-HP, Hôpital Louis Mourier, Colombes, France

${ }^{9}$ Bacteriology Laboratory, AP-HP, Hôpital Beaujon, Clichy, France

${ }^{10} \mathrm{AP}-\mathrm{HP}$, Hôpital Bichat-Claude Bernard, Medical Information Systems

Program (PMSI), Paris, France

${ }^{11}$ Infectious Diseases Department, AP-HP, Hôpital Bichat-Claude Bernard,

Paris, France

${ }^{12}$ Intensive Care Unit, AP-HP, Hôpital Louis Mourier, Colombes, France
}

Acknowledgements The authors thank the members of the infection control teams for their dedication: S Belorgey, W Guerinot, G Bendjelloul, I Garrigues and F Mignot.

Funding This research received no specific grant from any funding agency in the public, commercial or not-for-profit sectors.

Competing interests None declared.
Ethics approval Ethical committee of the Bichat-Claude Bernard Hospital group.

Provenance and peer review Not commissioned; externally peer reviewed.

Data sharing statement No additional data are available.

Open Access This is an Open Access article distributed in accordance with the Creative Commons Attribution Non Commercial (CC BY-NC 4.0) license, which permits others to distribute, remix, adapt, build upon this work noncommercially, and license their derivative works on different terms, provided the original work is properly cited and the use is non-commercial. See: http:// creativecommons.org/licenses/by-nc/4.0/

\section{REFERENCES}

1. Glasner C, Albiger B, Buist G, et al., European Survey on Carbapenemase-Producing Enterobacteriaceae (EuSCAPE) Working Group. Carbapenemase-producing Enterobacteriaceae in Europe: a survey among national experts from 39 countries, February 2013. Euro Surveill 2013;18:pii: 20525.

2. European Center for Disease Prevention And Control. Antimicrobial resistance interactive database (EARS-Net). 2012. http:// wwwecdceuropaeu/en/healthtopics/antimicrobial_resistance/ database/Pages/databaseaspx

3. Haut Conseil de la Santé Publique. Prévention de la transmission croisée des Bactéries Hautement Résistantes aux antibiotiques émergentes (BHRe). 2013 Juillet. http://www.hcsp.fr/explore.cgi/ avisrapportsdomaine?clefr $=372$

4. Birgand G, Armand-Lefevre L, Lepainteur M, et al. Introduction of highly resistant bacteria into a hospital via patients repatriated or recently hospitalized in a foreign country. Clin Microbiol Infect 2014;20:0887-90.

5. Birgand G, Ruimy R, Schwarzinger M, et al. Rapid detection of glycopeptide-resistant enterococci: impact on decision-making and costs. Antimicrob Resist Infect Control 2013;2:30.

6. Spence MR, Dammel T, Courser S. Contact precautions for methicillin-resistant Staphylococcus aureus colonization: costly and unnecessary? Am J Infect Control 2012;40:535-8.

7. Papia G, Louie M, Tralla A, et al. Screening high-risk patients for methicillin-resistant Staphylococcus aureus on admission to the hospital: is it cost effective? Infect Control Hosp Epidemiol 1999;20:473-7.

8. Ruppé E, Armand-Lefèvre L, Lolom I, et al. Development of a phenotypic method for detection of fecal carriage of OXA-48-producing enterobacteriaceae after incidental detection from clinical specimen. J Clin Microbiol 2011;49:2761-2.

9. Armand-Lefèvre L, Angebault C, Barbier F, et al. Emergence of imipenem-resistant Gram-negative bacilli in intestinal flora of intensive care patients. Antimicrob Agents Chemother 2013;57:1488-95

10. Agence Technique de l'Information sur l'Hospitalisation. Echelle nationale de coûts par GHM (en euros), Référentiel national de coûts. 2011. http://wwwatihsantefr/

11. Refaeilzadeh P, Tang L, Liu H. Cross-validation. Encyclopaedia Database Syst 2009:532-8 .

12. Daroukh A, Delaunay C, Bigot S, et al. Characteristics and costs of carbapenemase-producing enterobacteria carriers (2012/2013). Med Mal Infect 2014;44:321-6.

13. Escaut L, Bouam S, Frank-Soltysiak M, et al. Eradication of an outbreak of vancomycin-resistant Enterococcus (VRE): the cost of a failure in the systematic screening. Antimicrob Resist Infect Control 2013;2:18.

14. Armstrong-Evans M, Litt M, McArthur MA, et al. Control of transmission of vancomycin-resistant Enterococcus faecium in a long-term-care facility. Infect Control Hosp Epidemiol 1999;20:312-17.

15. Lloyd-Smith P, Younger J, Lloyd-Smith E, et al. Economic analysis of vancomycin-resistant enterococci at a Canadian hospital: assessing attributable cost and length of stay. J Hosp Infect 2013:85:54-9.

16. Cheah AL, Spelman T, Liew D, et al. Enterococcal bacteraemia: factors influencing mortality, length of stay and costs of hospitalization. Clin Microbiol Infect 2013;19:E181-9.

17. Carmeli Y, Eliopoulos G, Mozaffari E, et al. Health and economic outcomes of vancomycin-resistant enterococci. Arch Intern Med 2002;162:2223-8.

18. Shadel BN, Puzniak LA, Gillespie KN, et al. Surveillance for vancomycin-resistant enterococci: type, rates, costs, and implications. Infect Control Hosp Epidemiol 2006;27:1068-75. 
19. Lee T, Hacek D, Stroupe K, et al. Three surveillance strategies for vancomycin-resistant enterococci in hospitalized patients: detection of colonization efficiency and a cost effectiveness model. Infect Control Hosp Epidemiol 2005;26:39-46.

20. Muto C, Giannetta E, Durbin L, et al. Cost-effectiveness of perirectal surveillance cultures for controlling vancomycin-resistant Enterococcus. Infect Control Hosp Epidemiol 2002;23:429-35.

21. Montecalvo MA, Jarvis WR, Uman J, et al. Costs and savings associated with infection control measures that reduced transmission of vancomycin-resistant enterococci in an endemic setting. Infect Control Hosp Epidemiol 2001;22:437-42.

22. Robert J, Pantel A, Mérens A, et al., on behalf of ONERBA's Carbapenem Resistance Study Group. Incidence rates of carbapenemase-producing Enterobacteriaceae clinical isolates in France: a prospective nationwide study in 2011-12. J Antimicrob Chemother 2014;69:2706-12.

23. Fournier S, Brun-Buisson C, Jarlier V. Twenty years of antimicrobial resistance control programme in a regional multi hospital institution, with focus on emerging bacteria (VRE and CPE). Antimicrob Resist Infect Control 2012;1:9.

24. Fournier S, Brossier F, Fortineau N, et al. Long-term control of vancomycin-resistant Enterococcus faecium at the scale of a large multihospital institution: a seven-year experience. Euro Surveill 2012;17:pii: 20229

25. ECDC. Carbapenemase-producing bacteria in Europe. Interim results from the European survey on carbapenemase-producing
Enterobacteriaceae (EuSCAPE) project 2013. 2013. http://www. ecdc.europa.eu/en/publications/Publications/antimicrobial-resistancecarbapenemase-producing-bacteria-europe.pdf

26. ECDC. Point prevalence survey of healthcare-associated infections and antimicrobial use in European acute care hospitals 2011-2012. 2013. http://www.ecdc.europa.eu/en/publications/ publications/healthcare-associated-infections-antimicrobial-usepps.pdf

27. Ayraud-Thévenot S, Huart C, Mimoz O, et al. Control of multi-drug-resistant Acinetobacter baumannii outbreaks in an intensive care unit: feasibility and economic impact of rapid unit closure. J Hosp Infect 2012;82:290-2.

28. Kanerva M, Blom M, Tuominen U, et al. Costs of an outbreak of meticillin-resistant Staphylococcus aureus. J Hosp Infect 2007;66:22-8.

29. Garlantezec R, Bourigault C, Boles JM, et al. Investigation and management of an imipenem-resistant oxa-23 Acinetobacter baumannii outbreak in an intensive care unit. Med Mal Infect 2011:41:430-6.

30. Sagel U, Schulte B, Heeg P, et al. Vancomycin-resistant enterococci outbreak, Germany, and calculation of outbreak start. Emerg Infect Dis 2008;14:317-19.

31. Birgand G, Schwarzinger M, Perozziello A, et al. Prolonged hospital stay, an adverse effect of strict national policy for controlling the spread of highly resistant microorganisms. Infect Control Hosp Epidemiol 2014;35:1427-9. 